\title{
ABONOS ORGÁNICOS COMERCIALES, ESTIÉRCOLES LOCALES Y FERTILIZACIÓN QUIIMICA EN LA PRODUCCIÓN DE PLÁNTULA DE CHILE POBLANO
}

\author{
COMMERCIAL ORGANIC FERTILIZERS, LOCAL MANURES AND CHEMICAL \\ FERTILIZATION IN THE PRODUCTION OF POBLANO PEPPER SEEDLINGS
}

\author{
Patricia Acevedo-Alcalá, Javier Cruz-Hernández y Oswaldo R. Taboada-Gaytán*
}

${ }^{1}$ Colegio de Postgraduados, Campus Puebla, Santiago Momoxpan, Municipio de San Pedro Cholula, Puebla, México.

*Autor de correspondencia (toswaldo@colpos.mx)

\section{RESUMEN}

La producción de plántula de buena calidad depende en buena medida del tipo de sustrato utilizado, de la proporción en que se mezclen sus componentes, del tipo y dosis de fertilización aplicada y del aporte nutricional de cada componente para el desarrollo inicial de la plántula. El objetivo del presente estudio fue determinar la mejor combinación de abonos orgánicos comerciales (Solep y Fernatol), estiércoles locales (vacuno y ovino) y turba (peat moss), junto con fertilización química $\left(0.5,1.0\right.$ y $1.5 \mathrm{~g} \mathrm{~L}^{-1}$ de la fórmula 20N-18P-20K) para producir plántula de chile (Capsicum annuum L.) Poblano. Para determinar la calidad de las plántulas se registraron variables como el índice de esbeltez y de calidad de Dickson, la relación del peso seco de la parte aérea y peso seco de la raíz, entre otras. Se encontró que el abono orgánico comercial Solep, aplicado en proporción de $20 \%$ del volumen del sustrato produjo plántulas de buena calidad reflejada en mayor altura, diámetro y longitud de raíz, pero principalmente por los índices de esbeltez y calidad de Dickson. La aplicación de fertilizante químico a una concentración de $1.0 \mathrm{~g} \mathrm{~L}^{-1}$ de la fórmula 20-18-20 favoreció la obtención de plántulas de buena calidad. La proporción del abono orgánico comercial o estiércol local utilizado como componente de sustrato no debe exceder del $40 \%$ del volumen total de la mezcla final.

Palabras clave: Capsicum annuum L., componentes de sustrato, índice de calidad de Dickson, índice de esbeltez.

\section{SUMMARY}

Production of good quality seedlings largely depends on the type of substrate used, the proportion in which their components are mixed, the type and dose of fertilization applied and the nutritional contribution of each component for the initial development of the seedling. The objective of the present study was to determine the best combination of commercial organic fertilizers (Solep and Fernatol), local manures (cattle and sheep) and peat (peat moss), along with chemical fertilization $\left(0.5,1.0\right.$ and $1.5 \mathrm{~g} \mathrm{~L}^{-1}$ of the $20 \mathrm{~N}-18 \mathrm{P}-20 \mathrm{~K}$ formula) to produce Poblano pepper seedlings. To determine the quality of the seedlings, variables such as the slenderness and Dickson quality indexes, the ratio of the dry weight of the aerial part and the dry weight of the root, among others, were recorded. It was found that the commercial organic fertilizer Solep, applied in a proportion of $20 \%$ of the volume of the substrate produced seedlings of good quality reflected by larger height, diameter and root length, but mainly by the slenderness and Dickson quality indexes. The application of chemical fertilizer at a concentration of $1.0 \mathrm{~g} \mathrm{~L}^{-1}$ of the formula 20-18-20 favored the obtaining of good quality seedlings. The proportion of commercial organic fertilizer or local manure used as substrate components should not exceed $40 \%$ of volume of the final mixture.

Index words: Capsicum annuum, Dickson quality index, slenderness index, substrate components.

\section{INTRODUCCIÓN}

La producción de plántula de buena calidad es importante para los productores de diversas hortalizas o viveristas, pues esto mejora el establecimiento del cultivo en campo. Entre los factores que determinan la producción de plántulas de buena calidad están el mantener un estado nutricional apropiado por medio de la fertilización, proporcionar niveles adecuados de humedad a través del riego y mantener la sanidad mediante el control de plagas y prevención de enfermedades (Guzmán y Sánchez, 2003; Tuzel y Oztekin 2017), lo que permite obtener plántulas vigorosas y sanas. Un factor fundamental en la producción de plántula de calidad aceptable es el tipo de sustrato y las características propias del material que se utiliza con este propósito (García et al., 2011; Ortega-Martínez et al., 2010).

En la producción de plántulas se utilizan comúnmente sustratos comerciales debido a sus características fisicoquímicas; no obstante, la disponibilidad de las turbas en la actualidad cada vez es menor debido a que es un recurso natural no renovable (Tuzel et al., 2014, Tuzel y Oztekin, 2017), son de importación y con precios que se incrementan constantemente (Brito et al., 2015). Como alternativa a los sustratos comerciales existen diversos tipos de materiales orgánicos que se pueden utilizar para este fin y promover el crecimiento de las plántulas (González et al., 2014); para ello, es esencial que sean un medio de cultivo apropiado y que proporcionen los nutrientes necesarios para el buen desarrollo de éstas (Abid et al., 2018)

Las funciones de un buen sustrato para la producción 
de plántulas son proveer y mantener niveles adecuados de humedad para la semilla y posteriormente para la plántula, suministrar los nutrientes necesarios para el desarrollo de las plántulas, tener un buen intercambio gaseoso entre la atmósfera y el sustrato y actuar como soporte físico para la plántula (Rodríguez et al., 2010). Entre los tipos de sustrato que se pueden utilizar se encuentran los inertes como agrolitas o perlitas y materiales orgánicos como biosólidos (lodos de aguas residuales tratadas), residuos de cosecha, lombricompostas, compostas, estiércol de ganado y aves, abonos orgánicos y suelo, entre otros, en diferentes proporciones (García et al., 2011); adicionalmente, se pueden usar residuos de papel y sustratos agotados del cultivo de hongos, ya que después de un proceso de compostaje y maduración representan una alternativa al uso de los musgos comerciales (Jayasinghe et al., 2010). Estos materiales pueden ser de menor costo y de mayor disponibilidad para los productores de plántula en el ámbito local (Abid et al., 2018); por esta razón, la búsqueda de alternativas para utilizar diversos materiales orgánicos locales como sustratos o componentes de sustratos ha tenido un auge importante en las investigaciones a nivel mundial (Cruz-Crespo et al., 2013).

Por lo anterior, el objetivo de esta investigación fue determinar la mejor combinación de abono orgánico comercial (Solep y Fernatol), estiércol local (vacuno y ovino) y fertilización química $\left(0.5,1.0\right.$ y $1.5 \mathrm{~g} \mathrm{~L}^{-1}$ de la fórmula 2018-20) para producir plántulas de chile Poblano de buena calidad en la región de la Sierra Nevada de Puebla, bajo la premisa de que al menos una combinación de los factores bajo estudio permitirá formular un sustrato apropiado.

\section{MATERIALES Y MÉTODOS}

\section{Sitio experimental y material genético}

El experimento se realizó del 16 de marzo al 6 de mayo de 2017 bajo invernadero en las instalaciones de la Unidad Académica Regional Huejotzingo del Campus Puebla del Colegio de Postgraduados, en Huejotzingo, Puebla. El invernadero estuvo cubierto con plástico blanco Calibre 720 con 70 \% de transmisión de luz. Se utilizó semilla de chile Poblano de una variedad criolla local (TECAL-CP-16) cosechada en San Buenaventura Tecaltzingo, San Martín Texmelucan, Puebla en el ciclo 2016.

\section{Sustratos utilizados}

Los materiales utilizados como sustratos fueron: a) Solep, abono orgánico comercial de la empresa Soluciones Ecológicas de Puebla, Puebla, México; b) Fernatol, abono orgánico comercial de la empresa Fertilizante Natural obtenido por lombricomposteo, Puebla, México; c) estiércol vacuno, d) estiércol ovino disponibles localmente y colectados de pequeñas unidades de producción ganadera de San Nicolás de los Ranchos, Puebla; e) peat moss a base de musgo de origen canadiense, Sunshine Mezcla 3 grado profesional y f) vermiculita; los dos últimos se utilizan de manera comercial en la producción de plántulas.

La siembra se llevó a cabo el 16 de marzo de 2017 en charolas de poliestireno de 200 cavidades, en las que se depositaron dos semillas por cavidad. Inmediatamente después de la siembra y antes de cubrir la semilla con una ligera capa de sustrato se aplicó el fungicida Previcur ${ }^{\circledR} 1 \mathrm{~mL}$ $L^{-1}$ para prevenir posibles daños por hongos fitopatógenos; posteriormente, las charolas se apilaron y cubrieron con plástico transparente para mantener buenos niveles de humedad y temperatura. Dentro de las charolas cubiertas con plástico se colocó un datalogger (LogTag HAXO-8, Karlsruhe, Alemania) para registrar la temperatura y la humedad relativa durante la germinación y emergencia. La temperatura promedio dentro de las charolas cubiertas fue de $25.9{ }^{\circ} \mathrm{C}$ y un promedio de $53 \%$ de humedad relativa. A los 15 días después de la siembra las charolas se extrajeron de su envoltura plástica y se registraron las variables en las plántulas dentro del invernadero. A los 24 días después de la siembra se comenzó a aplicar el fertilizante Technigro ${ }^{\circledR}$ con la fórmula $20 \mathrm{~N}-18 \mathrm{P}-20 \mathrm{~K}$ tres veces por semana hasta el final del ciclo de la plántula; la concentración de la solución fue de 0.5, 1.0 y $1.5 \mathrm{~g} \mathrm{~L}^{-1}$ y el volumen aplicado fue hasta el límite de no ocasionar encharcamientos en las charolas. El Technigro® es un fertilizante químico soluble en agua disponible de manera comercial, que además aporta microelementos necesarios para el desarrollo de las plántulas. Con el fin de evitar daños ocasionados por hongos fitopatógenos, se hicieron aplicaciones de los fungicidas Previcur ${ }^{\circledR}$ o Prozycar ${ }^{\circledR}$ a concentración de $1 \mathrm{~mL} \mathrm{~L}^{-1}$ y $2 \mathrm{~g} \mathrm{~L}^{-1}$, respectivamente cada 15 días. La temperatura dentro del invernadero osciló entre 4.6 y $39.8^{\circ} \mathrm{C}$ y la humedad relativa de 5.9 a $100 \%$ durante el ciclo de la plántula.

\section{Tratamientos, diseño y unidad experimental}

El diseño experimental utilizado fue en bloques completos al azar con arreglo en parcelas divididas con cuatro repeticiones. La parcela grande consistió en la fertilización química $\left(0.5,1.0\right.$ y $1.5 \mathrm{~g} \mathrm{~L}^{-1}$ del fertilizante 20 18-20) con siete charolas de poliestireno; la parcela chica fue un grupo de 90 cavidades donde se incluyeron las proporciones de 20,40 y $60 \%$ de los materiales orgánicos evaluados en términos de volumen. Para los contenidos de clorofila se utilizó un diseño completamente al azar con tres repeticiones y como unidad experimental un grupo de cuatro plantas con competencia completa. En el Cuadro 
Cuadro 1. Descripción, pH, conductividad eléctrica, densidad aparente y porcentaje de humedad de las mezclas evaluadas.

\begin{tabular}{|c|c|c|c|c|c|}
\hline Tratamiento & Proporción del sustrato en volumen & $\mathrm{pH}$ & $\mathrm{CE}\left(\mathrm{mS} \mathrm{cm}^{-1}\right)$ & $\mathrm{DA}\left(\mathrm{g} \mathrm{cm}^{-3}\right)$ & $\% \mathrm{H}$ \\
\hline Sol 20 \% & 20-20-60 \% (Solep-Vermiculita-Peat moss) & 7.6 & 0.51 & 0.30 & 65.7 \\
\hline Sol $40 \%$ & 40-20-40 \% (Solep-Vermiculita-Peat moss) & 7.9 & 0.72 & 0.39 & 59.5 \\
\hline Sol 60 \% & 60-20-20 \% (Solep-Vermiculita-Peat moss) & 7.9 & 1.05 & 0.53 & 48.5 \\
\hline Fer $20 \%$ & 20-20-60 \% (Fernatol-Vermiculita-Peat moss) & 7.6 & 1.22 & 0.35 & 64.2 \\
\hline Fer $40 \%$ & 40-20-40 \% (Fernatol-Vermiculita-Peat moss) & 7.8 & 1.72 & 0.46 & 57.3 \\
\hline Fer $60 \%$ & 60-20-20\% (Fernatol-Vermiculita-Peat moss) & 7.9 & 1.99 & 0.52 & 52.4 \\
\hline EV $20 \%$ & 20-20-60 \% (Estiércol Vacuno-Vermiculita-Peat moss) & 7.6 & 0.89 & 0.29 & 63.0 \\
\hline EV $40 \%$ & 40-20-40 \% (Estiércol Vacuno-Vermiculita-Peat moss) & 7.9 & 1.21 & 0.45 & 59.0 \\
\hline EV $60 \%$ & 60-20-20 \% (Estiércol Vacuno-Vermiculita-Peat moss) & 8.1 & 1.65 & 0.65 & 50.8 \\
\hline EO $20 \%$ & 20-20-60 \% (Estiércol Ovino-Vermiculita-Peat moss) & 7.9 & 0.57 & 0.29 & 69.2 \\
\hline EO $40 \%$ & 40-20-40 \% (Estiércol Ovino-Vermiculita-Peat moss) & 8.2 & 0.77 & 0.37 & 65.5 \\
\hline EO $60 \%$ & 60-20-20 \% (Estiércol Ovino-Vermiculita-Peat moss) & 8.6 & 1.01 & 0.48 & 57.9 \\
\hline Testigo & 20-80 \% (Vermiculita-Peat moss) & 6.9 & 0.23 & 0.18 & 76.6 \\
\hline
\end{tabular}

CE: conductividad eléctrica, DA: densidad aparente y \% $\mathrm{H}$ : porcentaje de humedad.

1 se muestra la descripción, pH, conductividad eléctrica, densidad aparente y porcentaje de humedad de las mezclas evaluadas como sustratos. La aleatorización de bloques (repeticiones), parcelas grandes y parcelas chicas se realizó en el programa Statistical Analysis System 9.4 (SAS Institute, 2013).

\section{Variables de calidad}

Para el registro de estas variables se seleccionaron 10 plantas por unidad experimental con competencia completa. Las variables registradas fueron el porcentaje de emergencia (\% PE), que se obtuvo por conteo de las plantas emergidas cada tercer día; altura de planta final (AP), que se midió en $\mathrm{cm}$ con una regla cada $5 \mathrm{~d}$, AP corresponde a la última lectura registrada a los 52 días después de la siembra; longitud de raíz (LR), se midió en cm a los 52 días después de la siembra; diámetro de tallo final (Di), se midió en mm con un vernier digital Truper Stainless Steel cada $5 \mathrm{~d}$, a los 52 días después de la siembra; número final de hojas verdaderas; lecturas SPAD con medidor SPAD-502 (Tokio, Japón) (Konica Minolta, 1989) en dos hojas, una superior y una inferior y contrarias a su posición, para estimar la cantidad relativa de clorofila presente en las hojas; se determinó el contenido de clorofila a, b y total por extracción con acetona (Aguilar y Peña, 2006), para la determinación y lectura se utilizó una centrífuga (Hermle modelo Z 326 $\mathrm{K}$, Gosheim, Alemania) y un espectrofotómetro (ThermoElectron UV/V15, Waltham, Massachusetts, EUA) una semana antes de la cosecha de la plántula.

\section{Variables de acumulación de materia seca}

Se determinó el peso seco de brote (PSB) y peso seco de raíz (PSR) en g en una balanza analítica (Explorer ${ }^{\circledR}$ Pro Ohaus, Parsippany, New Jersey, EUA) después de secar las plántulas en un horno de Shel Lab modelo FX28 (Sheldon Manufacturing, Cornelius, Oregon, EUA). Adicionalmente, se calcularon el índice de esbeltez (IE) como la relación entre la altura y el diámetro del tallo, índice de calidad de Dickson (ICD) mediante la fórmula:

$$
I C D=P S T /(A P / D i+P S B / P S R)
$$

donde: PST es el peso seco total de la planta (g), PSB es el peso seco de la parte aérea (g), PSR es el peso seco de la raíz (g), AP es la altura de la planta $(\mathrm{cm})$ y Di el diámetro de la misma $(\mathrm{mm})$; se calculó también la relación del peso seco de la parte aérea y peso seco de la raíz (RPAR) (Piña y Arboleda, 2010), estabilidad del sustrato (Es) consiste en la retención del cepellón, clasificada como alta: $100 \%$ (3), media: más del $50 \%$ (2) y baja: menos del $50 \%$ (1) y la facilidad de extracción (Ex) es el desprendimiento de la plántula de la bandeja clasificada como fácil (1), media (2) y difícil de extraer (3) (González et al., 2014).

\section{Análisis estadístico}

La información se analizó estadísticamente por medio de análisis de varianza y comparación de medias de Tukey $(P \leq 0.05)$ con el programa Statistical Analysis System 9.4 
(SAS Institute, 2013).

\section{RESULTADOS Y DISCUSIÓN}

En el Cuadro 2 se muestran los cuadrados medios de las variables de calidad en plántulas de chile Poblano. Se presentaron diferencias estadísticas significativas ( $P \leq$ 0.001 ) en el $75 \%$ de las variables al considerar el efecto de la fertilización química (F); en los sustratos (S) se observaron diferencias en todas las variables evaluadas y en la interacción de $\mathrm{F} \times \mathrm{S}$ sólo en el $37.5 \%$ de las mismas. De manera general, las diferencias significativas se presentaron principalmente en las variables de altura, diámetro, número de hojas y peso seco de raíz. OrtegaMartínez et al. (2010) mencionan que el efecto de los sustratos en el desarrollo de las plántulas ocasiona diferencias estadísticas significativas en las variables relacionadas con el crecimiento y acumulación de materia seca, lo cual coincide con lo obtenido en esta investigación.

Los cuadrados medios de los índices de calidad en plántulas de chile Poblano se muestran en el Cuadro 3. Se observó que para repeticiones ( $R$ ) hubo diferencias significativas en todas las variables, con excepción de la estabilidad del sustrato, mientras que para fertilización química (F) sólo hubo diferencias estadísticas en tres variables (IE, RPAR y Ex). Los sustratos (S) fueron estadísticamente diferentes $(P \leq 0.001)$ para todos los índices calculados y variables registradas; para la interacción de $\mathrm{F} \times \mathrm{S}$ sólo la estabilidad del sustrato fue estadísticamente diferente $(P \leq 0.001)$. Se observaron diferencias significativas en el índice de esbeltez, relación del peso seco de la parte aérea/raíz y en la estabilidad del sustrato.
La calidad de la plántula está asociada a la combinación de sustratos y la aplicación de fertilización química (García et al., 2011), tal como ocurrió en el presente estudio. La interacción de $\mathrm{F} \times \mathrm{S}$ presentó diferencias significativas en la variable estabilidad del sustrato, lo que indica que la combinación de sustratos y la fertilización química influyen en esta variable, que causó en algunos casos el desprendimiento de la raíz y a su vez problemas al momento del transplante (González et al., 2014). Los dos índices de calidad evaluados no fueron afectados por la interacción de F × S.

En el Cuadro 4 se presenta la comparación de medias de las variables de calidad en plántulas para las fuentes de variación de fertilización (F) y sustratos (S). El testigo (turba) y los tratamientos al 20 y $40 \%$ de los abonos o estiércoles alcanzaron resultados semejantes en las variables de altura de planta y longitud de raíz; esto significa que en estos indicadores no es necesario utilizar materiales orgánicos importados y que éstos se pueden substituir por abonos orgánicos de origen nacional o, inclusive, por estiércoles disponibles localmente para obtener plántulas de calidad.

En las variables de PE, Di y número de hojas se observaron resultados similares entre el testigo y los porcentajes al 20, 40 y $60 \%$ del abono Solep y el estiércol ovino. En las variables de PSB y PSR el abono Solep, Fernatol y el estiércol vacuno al $20 \%$ produjeron la mayor acumulación de materia seca, pero con valores similares al testigo. El estiércol de ovino resultó con la menor acumulación de materia seca en brote al 20, 40 y 60 \% en comparación con el testigo. Adicionalmente, en todas las variables para los tratamientos que incluyeron una proporción de $60 \%$ del abono Fernatol y del estiércol vacuno se obtuvieron

Cuadro 2. Cuadrados medios de variables de calidad en plántulas de chile Poblano producidas con diferentes sustratos en invernadero.

\begin{tabular}{|c|c|c|c|c|c|c|c|c|c|}
\hline \multirow{2}{*}{$\begin{array}{l}\text { Fuente de } \\
\text { variación }\end{array}$} & \multicolumn{9}{|c|}{ Variable } \\
\hline & gl & PE & AP & Di & $\mathrm{Ho}$ & $\mathrm{ClS}$ & LR & PSB & PSR \\
\hline Repeticiones (R) & 3 & 212.33 ns & $13.56 * \star \star$ & 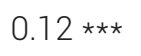 & $1.25 * \star \star$ & $14.59 \mathrm{~ns}$ & $3.86 \mathrm{~ns}$ & $0.10 \mathrm{~ns}$ & $0.18 * * *$ \\
\hline Fertilización (F) & 2 & 3.89 ns & $29.71 * * *$ & $0.44 * * *$ & $6.74 * * *$ & $309.54 * * *$ & $11.05 * * *$ & $1.74 * * \star$ & 0.10 * \\
\hline Error a & 6 & 123.46 & 0.76 & 0.08 & 0.17 & 14.09 & 1.70 & 0.03 & 0.05 \\
\hline Sustratos (S) & 12 & $8302.62 * * *$ & $47.38 * * *$ & $0.47 * * *$ & $6.40 * * *$ & $172.02 * * \star$ & $20.83 * * *$ & $1.65 * * \star$ & $0.47 * * *$ \\
\hline$F \times S$ & 24 & $21.88 \mathrm{~ns}$ & $1.47 * \star *$ & $0.04 *$ & $0.18 \mathrm{~ns}$ & $18.21 * * \star$ & $1.59 \mathrm{~ns}$ & $0.11 * * *$ & $0.03 \mathrm{~ns}$ \\
\hline Error b & 108 & 97.94 & 0.58 & 0.02 & 0.13 & 7.16 & 1.46 & 0.04 & 0.03 \\
\hline CV (\%) & & 11.56 & 8.37 & 7.17 & 5.06 & 7.02 & 12.28 & 14.93 & 27.16 \\
\hline
\end{tabular}

gl: grados de libertad, PE: porcentaje de emergencia, AP. altura de planta final, Di: diámetro de tallo final, Ho: número de hojas final, CIS: lecturas SPAD, LR: longitud de raíz, PSB: peso seco de brote, PSR: peso seco de raíz, ns: diferencia no significativa, * y ***: significancia estadística a P $\leq 0.05$ y P $\leq$ 0.001, respectivamente, CV: coeficiente de variación. 
resultados inferiores en comparación con el testigo y los demás tratamientos.

Las mejores características agronómicas en los tratamientos así resultantes podría deberse a un mejor aporte de nutrientes y a una retención de humedad más apropiada (Berrospe-Ochoa et al., 2012). Al utilizar un sustrato integrado por diferentes componentes es posible que ocurra una mayor disponibilidad de nutrientes, así como sinergismo entre los mismos, lo que provoca mejoras fisiológicas en las plántulas (Gómez-Merino et al., 2013). El mejor desarrollo o crecimiento de las plántulas se atribuye a la presencia de características físicas apropiadas como aireación y absorción (González et al., 2014) y a características químicas adecuadas en términos de pH y CE de los sustratos (López-Baltazar et al., 2013), lo que indica una mayor humificación de los compuestos orgánicos y hace que estos materiales sean apropiados para su uso como sustratos o componentes de sustratos.

En la fertilización química no se observaron diferencias en las variables PE, AP, Di, Ho, CIS, PSB y PSR tras aplicar 1.0 ó $1.5 \mathrm{~g} \mathrm{~L}^{-1}$ de fertilizante, lo que indica que no es necesario aplicar concentraciones altas de fertilizantes químicos cuando el sustrato a utilizar incluye abonos orgánicos comerciales o estiércoles locales, mismos que aportan nutrientes esenciales para el buen desarrollo de las plántulas en sus primeras etapas. En longitud de raíz no se observaron diferencias estadísticas significativas al aplicar el fertilizante químico a $0.5 \circ 1.0 \mathrm{~g} \mathrm{~L}^{-1}$; sin embargo, los tratamientos con $1.5 \mathrm{~g} \mathrm{~L}^{-1}$ presentaron menor longitud de raíz, lo que indica, nuevamente, que no es estrictamente necesario utilizar altas concentraciones de fertilizantes químicos. Por lo tanto, bajo un buen manejo de la fertilización, incluyendo elementos mayores y algunos microelementos, podrán obtenerse plántulas de buena calidad (Gómez-Merino et al., 2013). Los resultados indican que los abonos o estiércoles se pueden combinar con otros sustratos en proporciones de 20 y $40 \%$ con fertilización de 1.0 ó $1.5 \mathrm{~g} \mathrm{~L}^{-1}$.

La comparación de medias de los índices de calidad se muestra en el Cuadro 5. Los valores de índice de esbeltez menores de seis se asocian a plantas de buena calidad (Romero-Arenas et al., 2012), mientras que los valores superiores a 8 indican plántula de baja calidad (Sáenz et al., 2010). En el índice de calidad de Dickson, valores mayores a 0.5 indican plántulas de buena calidad, entre 0.5 y 0.2 calidad media, mientras que valores menores a 0.2 se asignan a plántulas de baja calidad (Sáenz et al., 2010). Este tipo de índices pueden variar dependiendo de la especie, el tipo y proporción del sustrato, el volumen del alveolo y principalmente el momento en que se determinan las variables (Faria et al., 2016); valores aceptables de los mismos indican que la plántula tiene un balance en términos de crecimiento de la parte aérea y radicular, lo cual garantiza un buen desarrollo de la planta (RomeroArenas et al., 2012).

Los resultados obtenidos en IE indican que todos los tratamientos presentaron buena calidad, con valores menores de 6, mientras que en ICD el mejor resultado se consiguió con el tratamiento Solep $40 \%$, con un valor cercano al 0.5 , indicador de alta o buena calidad; por el contrario, los demás tratamientos se clasifican como plántula de calidad media, con excepción de los tratamientos con abono Fernatol y el estiércol vacuno al $60 \%$, los cuales presentan una mala o baja calidad, pues

Cuadro 3. Cuadrados medios de índices de calidad calculados en plántulas de chile Poblano producidas con diferentes componentes de sustrato en invernadero.

\begin{tabular}{lclllll}
\hline \multirow{2}{*}{ Fuente de variación } & \multicolumn{7}{c}{ Índices de calidad } \\
\cline { 2 - 7 } & $\mathrm{gl}$ & $\mathrm{IE}$ & $\mathrm{ICD}$ & $\mathrm{RPAR}$ & Es & Ex \\
\hline Repeticiones (R) & 3 & $5.85 * * *$ & $0.06 * * *$ & $1.19 * *$ & $0.04 \mathrm{~ns}$ & $1.66 * * *$ \\
Fertilización (F) & 2 & $1.33 * * *$ & $0.01 \mathrm{~ns}$ & $10.35 * * *$ & $0.37 * * *$ & $0.20 \mathrm{~ns}$ \\
Error a & 6 & 0.16 & 0.01 & 0.33 & 0.07 & 0.29 \\
Sustratos (S) & 12 & $4.88 * * *$ & $0.07 * * *$ & $1.00 * * *$ & $2.69 * * *$ & $4.16 * * *$ \\
F×S & 24 & $0.14 \mathrm{~ns}$ & $0.01 \mathrm{~ns}$ & $0.31 \mathrm{~ns}$ & $0.20 * * *$ & $0.24 \mathrm{~ns}$ \\
Error b & 108 & 0.13 & 0.00 & 0.34 & 0.07 & 0.22 \\
CV (\%) & & 8.62 & 22.52 & 24.79 & 9.59 & 27.75 \\
\hline
\end{tabular}

gl: grados de libertad, IE: índice de esbeltez, ICD: índice de calidad de Dickson, RPAR: relación del peso seco de la parte aérea/peso seco de la raíz, Es: estabilidad del sustrato, Ex: facilidad de extracción de la plántula, ns: diferencia no significativa, ** y ***: diferencias significativas a P $\leq 0.01$ y P $\leq$ 0.001, respectivamente, CV: coeficiente de variación 
Cuadro 4. Comparación de medias de los tratamientos bajo estudio para variables de calidad en plántulas de chile Poblano.

\begin{tabular}{|c|c|c|c|c|c|c|c|c|}
\hline \multirow{2}{*}{ Factores de estudio } & \multicolumn{8}{|c|}{ Variables } \\
\hline & PE (\%) & $\mathrm{AP}(\mathrm{cm})$ & Di (mm) & $\mathrm{Ho}$ & $\mathrm{ClS}$ & $\mathrm{LR}(\mathrm{cm})$ & PSB & PSR \\
\hline \multicolumn{9}{|c|}{ Sustratos } \\
\hline Sol-20 \% & $97.91 \mathrm{a}$ & 10.58 a & $2.22 \mathrm{a}$ & $7.38 \mathrm{a}$ & 40.28 abc & $10.46 a b$ & $1.51 \mathrm{abcd}$ & $0.77 a b$ \\
\hline Sol-40 \% & $93.84 a$ & $10.27 \mathrm{ab}$ & $2.34 \mathrm{a}$ & $7.45 a$ & 39.85 abc & $10.61 \mathrm{ab}$ & $1.58 \mathrm{abc}$ & $0.89 a$ \\
\hline Sol-60 \% & $88.61 \mathrm{a}$ & $8.94 \mathrm{c}$ & $2.22 \mathrm{a}$ & $7.16 \mathrm{a}$ & $41.92 \mathrm{ab}$ & $9.36 \mathrm{bc}$ & 1.38 bcde & $0.66 \mathrm{abc}$ \\
\hline Fer-20 \% & $98.51 \mathrm{a}$ & 10.32 a & $2.37 \mathrm{a}$ & $7.29 \mathrm{a}$ & $39.50 \mathrm{abcd}$ & $10.52 a b$ & $1.56 \mathrm{abc}$ & $0.71 \mathrm{abc}$ \\
\hline Fer-40 \% & 96.48 a & 9.26 bc & $2.20 \mathrm{a}$ & $7.25 \mathrm{a}$ & $40.25 a b c$ & $10.65 a b$ & 1.32 cde & 0.62 bc \\
\hline Fer-60 \% & $43.65 b$ & $5.21 \mathrm{~d}$ & $1.82 \mathrm{~b}$ & $5.75 b$ & $33.95 \mathrm{e}$ & $6.25 d$ & $0.70 \mathrm{f}$ & $0.25 d$ \\
\hline EV-20 \% & $96.94 \mathrm{a}$ & 10.36 a & $2.32 \mathrm{a}$ & $7.38 \mathrm{a}$ & $39.09 \mathrm{bcd}$ & $10.48 a b$ & $1.59 a b$ & $0.78 a b$ \\
\hline EV-40 \% & 95.18 a & $8.64 \mathrm{c}$ & $2.16 \mathrm{a}$ & $7.20 \mathrm{a}$ & 37.05 cde & $10.12 a b$ & $1.17 \mathrm{e}$ & $0.53 c$ \\
\hline EV-60 \% & $13.00 \mathrm{c}$ & $4.69 \mathrm{~d}$ & $1.71 \mathrm{~b}$ & $5.12 \mathrm{c}$ & $28.23 f$ & $8.01 \mathrm{c}$ & $0.45 f$ & $0.19 d$ \\
\hline EO-20 \% & $98.42 \mathrm{a}$ & 10.34 a & $2.26 \mathrm{a}$ & $7.24 \mathrm{a}$ & $38.65 \mathrm{bcd}$ & $10.41 \mathrm{ab}$ & $1.49 \mathrm{bcd}$ & $0.63 \mathrm{bc}$ \\
\hline EO-40 \% & 98.79 a & $10.19 a b$ & $2.26 \mathrm{a}$ & $7.45 a$ & $38.04 \mathrm{~cd}$ & $9.97 \mathrm{ab}$ & $1.45 \mathrm{bcd}$ & 0.62 bc \\
\hline EO-60 \% & 94.81 a & $8.73 c$ & $2.16 \mathrm{a}$ & $7.30 \mathrm{a}$ & $36.10 \mathrm{de}$ & $10.05 a b$ & $1.25 \mathrm{de}$ & $0.59 \mathrm{bc}$ \\
\hline Testigo (turba) & $96.35 \mathrm{a}$ & $10.85 a$ & $2.30 \mathrm{a}$ & $7.51 \mathrm{a}$ & $42.80 \mathrm{a}$ & $11.04 \mathrm{a}$ & $1.76 \mathrm{a}$ & $0.75 \mathrm{abc}$ \\
\hline DSH (0.05) & 13.68 & 1.05 & 0.21 & 0.49 & 3.70 & 1.67 & 0.27 & 0.23 \\
\hline \multicolumn{9}{|c|}{ Fertilización química } \\
\hline Fert-0.5 & 85.81 a & $8.25 b$ & $2.07 \mathrm{~b}$ & $6.62 b$ & $35.4 b$ & $10.05 \mathrm{a}$ & $1.12 b$ & $0.65 a$ \\
\hline Fert-1.0 & 85.65 a & $9.39 a$ & $2.23 \mathrm{a}$ & $7.23 \mathrm{a}$ & $38.91 \mathrm{a}$ & $10.16 \mathrm{a}$ & $1.38 \mathrm{a}$ & $0.63 a b$ \\
\hline Fert-1.5 & 85.27 a & $9.68 \mathrm{a}$ & $2.24 \mathrm{a}$ & $7.26 \mathrm{a}$ & 40.08 a & $9.31 b$ & $1.47 \mathrm{a}$ & $0.59 b$ \\
\hline DSH (0.05) & 13.68 & 1.05 & 0.21 & 0.49 & 3.70 & 1.67 & 0.27 & 0.23 \\
\hline
\end{tabular}

Medias con la misma letra en las columna dentro de cada factor de variación son iguales estadísticamente (Tukey, 0.05). Sol: Solep, Fer: Fernatol, EV: estiércol vacuno, EO: estiércol ovino, Fert: fertilización a 0.5, 1.0 y $1.5 \mathrm{~g} \mathrm{~L}^{-1}$, PE: porcentaje de emergencia, AP. altura de planta final, Di: diámetro de tallo final, Ho: número de hojas final, CIS: lecturas SPAD, LR: longitud de raíz, PSB: peso seco de brote, PSR: peso seco de raíz, DSH: diferencia significativa honesta.

los valores obtenidos son menores de 0.2. En la variable de RPAR, el valor superior se observó en el tratamiento con abono Fernatol $60 \%$ y el valor inferior se presentó con el abono Solep $40 \%$. Las plántulas obtenidas con el abono Solep no excedieron el valor de 2.5 en RPAR, lo que indica que éstas pueden sobrevivir al trasplante en campo, ya que se evita que la traspiración sea menor que la capacidad de absorción de agua, según lo reportado por (Reyes-Reyes et al., 2005). En las variables de estabilidad y extracción de la plántula de la charola se observó que en la mayoría de los tratamientos, con excepción del abono Fernatol y estiércol vacuno al $60 \%$, al extraer las plántulas del alveolo se retiraba más del $90 \%$ del sustrato, lo cual es considerado como una alta estabilidad y una extracción de fácil a media.
La facilidad de extracción y la estabilidad de los tratamientos pudo deberse a que presentan mejores características físicas, al no compactarse y teniendo una cohesión suficiente para no desprender la raíz del cepellón y así no causar problemas en la raíz al momento del trasplante (González et al., 2014).

En el efecto de la fertilización química, las variables ICD y Ex no presentaron diferencias significativas, mientras que la variable IE no presentó diferencias al aplicar $1.0 \mathrm{O}$ $1.5 \mathrm{~g} \mathrm{~L}^{-1}$; en la variable RPAR se presentaron diferencias al aplicar alguna de las tres concentraciones de fertilización. Los resultados indicaron que el utilizar el abono orgánico comercial Solep en proporciones de 20 y $40 \%$ en combinación con una concentración de fertilización intermedia $\left(1 \mathrm{~g} \mathrm{~L}^{-1}\right)$ es suficiente para la producción de plántulas de buena calidad. 
Cuadro 5. Comparación de medias de los tratamientos bajo estudio para los índices de calidad evaluados en plántulas de chile Poblano.

\begin{tabular}{|c|c|c|c|c|c|c|}
\hline \multirow{2}{*}{ Factores de estudio } & \multicolumn{6}{|c|}{ Índices de calidad } \\
\hline & & IE & ICD & RPAR & Es (1-3) & Ex (1-3) \\
\hline \multicolumn{7}{|l|}{ Sustratos } \\
\hline Sol-20 \% & & $4.78 \mathrm{a}$ & $0.34 a b$ & $2.07 \mathrm{~b}$ & $2.91 \mathrm{a}$ & $1.16 \mathrm{~cd}$ \\
\hline Sol-40 \% & & $4.38 \mathrm{abc}$ & $0.40 \mathrm{a}$ & $1.88 \mathrm{~b}$ & $3.00 \mathrm{a}$ & $1.25 \mathrm{bcd}$ \\
\hline Sol-60 \% & & $4.04 \mathrm{c}$ & $0.33 a b$ & $2.36 a b$ & $3.00 \mathrm{a}$ & $1.83 \mathrm{~b}$ \\
\hline Fer-20 \% & & $4.39 a b c$ & $0.35 a b$ & $2.25 b$ & $3.00 \mathrm{a}$ & $1.33 \mathrm{bcd}$ \\
\hline Fer-40 \% & & $4.21 \mathrm{bc}$ & $0.31 a b$ & $2.18 b$ & $2.91 \mathrm{a}$ & 1.66 bcd \\
\hline Fer-60 \% & & $2.86 \mathrm{~d}$ & $0.17 \mathrm{c}$ & $3.10 \mathrm{a}$ & $1.50 \mathrm{c}$ & $3.00 \mathrm{a}$ \\
\hline EV-20 \% & & $4.46 \mathrm{abc}$ & $0.36 a b$ & $2.17 \mathrm{~b}$ & $3.00 \mathrm{a}$ & $1.58 \mathrm{bcd}$ \\
\hline$E V-40 \%$ & & $4.04 \mathrm{c}$ & $0.27 b$ & $2.33 a b$ & $2.91 \mathrm{a}$ & 1.75 bc \\
\hline EV-60 \% & & $2.75 d$ & $0.12 \mathrm{c}$ & $2.56 a b$ & $2.00 \mathrm{~b}$ & $2.83 \mathrm{a}$ \\
\hline EO-20 \% & & $4.56 a b$ & $0.31 \mathrm{ab}$ & $2.41 \mathrm{ab}$ & $3.00 \mathrm{a}$ & $1.58 \mathrm{bcd}$ \\
\hline EO-40 \% & & $4.50 \mathrm{abc}$ & $0.30 \mathrm{~b}$ & $2.45 a b$ & $3.00 \mathrm{a}$ & $1.33 \mathrm{bcd}$ \\
\hline EO-60\% & & $4.04 \mathrm{c}$ & $0.30 b$ & $2.23 \mathrm{~b}$ & $3.00 \mathrm{a}$ & 1.66 bcd \\
\hline Testigo (turba) & & $4.69 a b$ & $0.35 a b$ & $2.41 \mathrm{ab}$ & $3.00 \mathrm{a}$ & $1.08 d$ \\
\hline DSH (0.05) & & 0.49 & 0.09 & 0.80 & 0.37 & 0.65 \\
\hline \multicolumn{7}{|l|}{ Fertilización química } \\
\hline Fert-0.5 & & $3.96 b$ & $0.30 \mathrm{a}$ & $1.87 \mathrm{C}$ & $2.73 b$ & $1.76 \mathrm{a}$ \\
\hline Fert-1.0 & & $4.16 \mathrm{a}$ & $0.31 \mathrm{a}$ & $2.39 b$ & $2.88 \mathrm{a}$ & $1.67 \mathrm{a}$ \\
\hline Fert-1.5 & & $4.27 \mathrm{a}$ & $0.29 a$ & $2.76 \mathrm{a}$ & $2.75 b$ & $1.65 \mathrm{a}$ \\
\hline DSH (0.05) & & 0.16 & 0.03 & 0.27 & 0.12 & 0.21 \\
\hline \multicolumn{7}{|c|}{$\begin{array}{l}\text { Medias con la misma letra en las columna dentro de cada factor de variación son iguales estadísticamente (Tukey, 0.05). Sol: Solep, Fer: Fernatol, E } \\
\text { estiércol vacuno, EO: estiércol ovino, Fert: fertilización a } 0.5,1.0 \text { y } 1.5 \mathrm{~g} \text { L-1, IE: índice de esbeltez, ICD: índice de calidad de Dickson, RPAR: relación d } \\
\text { peso seco de la parte aérea/peso seco de la raíz, Es: estabilidad del sustrato, Ex: facilidad de extracción de la plántula, DSH: diferencia significati } \\
\text { honesta; }\end{array}$} \\
\hline \multicolumn{7}{|c|}{$\begin{array}{l}\text { Cuadro 6. Cuadrados medios de contenidos de clorofila a, b y total en plántulas de chile Poblano producidas con diferente } \\
\text { sustratos en invernadero. }\end{array}$} \\
\hline \multirow{2}{*}{ Fuente de Variación } & \multirow{2}{*}{ gl } & & \multicolumn{4}{|c|}{ Clorofila } \\
\hline & & & a & & & Total \\
\hline Fertilización (F) & 2 & & $0.12 * * \star$ & 1.7 & & $14.42 \star \star \star$ \\
\hline Sustratos (S) & 12 & & $0.58 * * *$ & 0.7 & & $63.27 * * \star$ \\
\hline$F \times S$ & 24 & & $0.31 * * \star$ & 0.2 & & $44.59 * \star \star$ \\
\hline Error & 78 & & 0.0177 & 0.0 & & 2.4946 \\
\hline CV (\%) & & & 4.99 & 10.2 & & 5.16 \\
\hline
\end{tabular}

gl: grados de libertad, a: clorofila a; b: clorofila b; Total: clorofila total, ***: significancia a P $\leq 0.001$, CV: coeficiente de variación. 
Los cuadrados medios de las cantidades de clorofila a, b y total en las plántulas de chile Poblano se presentan en el Cuadro 6. Se obtuvieron diferencias estadísticas significativas $(P \leq 0.001)$ para los contenidos de clorofila a, b y total entre las dosis aplicadas de fertilización química, los sustratos evaluados y la interacción $\mathrm{F} \times \mathrm{S}$. Estas diferencias se deben a que el contenido de $\mathrm{N}$ en las hojas está directamente relacionado con el contenido de clorofila y la capacidad fotosintética de la planta (Castro y Blanco, 2018); es decir, existe un efecto directo sobre la cantidad de pigmentos fotosintéticos producidos por las plántulas como consecuencia de las diferentes concentraciones de abonos y estiércoles y por las diferentes concentraciones de fertilización química que se aplicaron. El contenido de clorofila está asociado con el color verde de las hojas debido a que el $\mathrm{N}$ es necesario para la síntesis de la clorofila (Betancourt-Olvera et al., 2005; Rincón y Ligarreto, 2010); además, se relaciona con el estado nutricional y el establecimiento y sobrevivencia de las plántulas en campo debido a que el potencial del sistema fotosintético empieza a convertir la energía lumínica en energía química, el N excedente formará parte de los compuestos de reserva, lo que ayuda a la supervivencia, establecimiento, aumento de la tasa fotosintética, acumulación de la biomasa y aumento del rendimiento del cultivo (Gómez-Merino et al., 2013; Rincón y Ligarreto, 2010).

En el Cuadro 7 se muestran las comparaciones de medias para los índices de calidad en plántulas. Se observaron diferencias entre tratamientos para clorofila a, b y total. El tratamiento con abono orgánico comercial Fernatol $20 \%$ presentó un valor superior en contenido de clorofila a y total, mientras que el tratamiento con el abono orgánico comercial Solep 20 \% tuvo el mayor contenido de clorofila b. Las menores cantidades de clorofila a se obtuvieron con el estiércol ovino $20 \%$, mientras que la menor cantidad de clorofila b y total resultó al aplicar el estiércol vacuno $60 \%$. Este comportamiento, ocasionado por la alta concentración de abonos orgánicos en las mezclas evaluadas se reflejó en la menor altura de planta en aquellos tratamientos que incluyeron $60 \%$ de estos materiales en los sustratos (Cuadro 4). En la fertilización química se observó que el mayor contenido de clorofila se obtuvo con $1.5 \mathrm{~g} \mathrm{~L}^{-1}$ de fertilizante 20-18-20. Esta dosis provoca que las hojas contengan más $\mathrm{N}$ y con ello sean capaces de sintetizar una mayor cantidad de clorofila y aumentar la acumulación de biomasa (Gómez-Merino et al., 2013), representada por una mayor altura de planta, diámetro de tallo y número de hojas (Cuadro 4). Por lo tanto, los abonos Fernatol y Solep al 20 \%, en combinación con $1.5 \mathrm{gL}^{-1}$ de fertilización, aumentaron el contenido de $\mathrm{N}$ y ésto a su vez el contenido de clorofila en las hojas.

\section{CONCLUSIONES}

El abono orgánico comercial Solep en proporción de $20 \%$ es recomendable como componente del sustrato para producir plántulas de buena calidad. La fertilización química en concentración intermedia $\left(1.0 \mathrm{~g} \mathrm{~L}^{-1}\right)$ produce plántulas de buena calidad; por lo tanto, no es necesario aplicar altas cantidades de fertilizantes químicos para producir plántulas de calidad cuando se incluyen estiércoles locales o abonos orgánicos comerciales a una proporción no mayor del 40 \% como componentes del sustrato.

\section{AGRADECIMIENTOS}

Al Colegio de Postgraduados y al CONACYT por el apoyo económico para realizar la investigación. A la unidad de Prestadores de Servicios Profesionales (PSP) de la Unidad Académica Regional Huejotzingo del Campus Puebla.

\section{BIBLIOGRAFÍA}

Abid W., S. Magdich, I. B. Mahmoud, K. Medhioub and E. Ammar (2018) Date palm wastes co-composted product: an efficient substrate for tomato (Solanum lycopercicum L.) seedling production. Waste and Biomass Valorization 9:45-55, https://doi.org/10.1007/ s12649-016-9767-y

Aguilar B. G y C. B. Peña V. (2006) Alteraciones fisiológicas provocadas por sequía en nopal (Opuntia ficus-indica). Revista Fitotecnia Mexicana 29:231-237.

Berrospe-Ochoa E. A., V. M. Ordaz-Chaparro, M. N. Rodríguez-Mendoza y R. Quintero-Lizaola (2012) Cachaza como sustrato para la producción de plántula de tomate. Revista Chapingo Serie Horticultura 18:141-156.

Betancourt-Olvera M., M. N. Rodríguez-Mendoza, M. Sandoval-Villa y E. A. Gaytán-Acuña (2005) Fertilización foliar una herramienta en el desarrollo del cultivo de Lilium cv. Stargazer. Revista Chapingo Serie Horticultura 11:371-378.

Brito L. M., M. Reis, I. Mourão and J. Coutinho (2015) Use of acacia waste compost as an alternative component for horticultural substrates. Communications in Soil Science and Plant Analysis 46:1814-1826, https://doi.org/10.1080/00103624.2015.10598 43

Castro Y. y E. L. Blanco (2018) Estimación del contenido de clorofila y nitrógeno en plantas de pimentón inoculadas con bacterias rizosféricas. Revista Científica UNET Agropecuaria 30:105-112.

Cruz-Crespo E., A. Can-Chulim, M. Sandoval-Villa, R. Bugarín-Montoya A. Robles-Bermúdez y P. Juárez-López (2013) Sustratos en la horticultura. Revista Bio Ciencias 2:17-26, https://doi. org/10.15741/revbio.02.02.03

Faria J. C. T., M. V. W. Caldeira, W. M. Delarmelina e R. L. F. Rocha (2016) Substratos alternativos na produção de mudas de Mimosa setosa Benth. Ciência Florestal 26:1075-1086, https://doi. org/10.5902/1980509824996

García M. C., O. R. Taboada G., H. López S., P. A. López, G. Mora A. y B. Tlapal B. (2011) Calidad de plántulas de chile 'poblano' en la Sierra Nevada de Puebla, México. Revista Fitotecnia Mexicana 34:115-121.

Gómez-Merino F. C., L. I. Trejo-Téllez, J. C. García-Albarado y V. MoralesRamos (2013) Lulo (Solanum quiroense Lamarck.) como nuevo elemento del paisaje en México: germinación y crecimiento en sustratos orgánicos. Revista Mexicana de Ciencias Agrícolas 5:877-887, https://doi.org/10.29312/remexca.v0i5.1293 
Cuadro 7. Comparación de medias de los contenidos de clorofila a, b y total en plántulas de chile Poblano producidas con diferentes sustratos en invernadero.

\begin{tabular}{|c|c|c|c|}
\hline \multirow{2}{*}{ Factores de estudio } & \multicolumn{3}{|c|}{ Clorofila $\left(\mathrm{mg} \mathrm{L}^{-1}\right)$} \\
\hline & a & $b$ & $\mathrm{~T}$ \\
\hline \multicolumn{4}{|c|}{ Sustratos } \\
\hline Sol-20 \% & $2.75 a b$ & $1.61 \mathrm{a}$ & 30.58 bc \\
\hline Sol-40 \% & $2.86 a b$ & $1.34 \mathrm{bc}$ & $32.69 a b$ \\
\hline Sol-60 \% & $2.77 a b$ & $1.52 a b$ & $31.09 a b$ \\
\hline Fer-20 \% & $2.90 \mathrm{a}$ & $1.20 \mathrm{~cd}$ & $33.55 \mathrm{a}$ \\
\hline Fer-40 \% & $2.81 \mathrm{ab}$ & $1.15 \mathrm{~cd}$ & $32.64 a b$ \\
\hline Fer-60 \% & $2.64 \mathrm{bc}$ & $1.06 \mathrm{~d}$ & $30.73 a b c$ \\
\hline$E V-20 \%$ & $2.83 a b$ & $1.49 a b$ & $31.88 a b$ \\
\hline$E V-40 \%$ & $2.73 a b$ & $1.34 \mathrm{bc}$ & $31.05 a b$ \\
\hline EV-60 \% & $1.94 \mathrm{~d}$ & $0.59 \mathrm{e}$ & $23.06 d$ \\
\hline EO-20 \% & $2.42 \mathrm{c}$ & $0.99 d$ & $28.10 \mathrm{C}$ \\
\hline EO-40 \% & $2.61 \mathrm{bc}$ & $1.01 \mathrm{~d}$ & $30.46 \mathrm{bc}$ \\
\hline EO-60 \% & $2.63 \mathrm{bc}$ & $1.09 \mathrm{~d}$ & $30.47 \mathrm{bc}$ \\
\hline Testigo (Peat moss) & $2.83 a b$ & $1.49 a b$ & $31.98 a b$ \\
\hline DSH (0.05) & 0.24 & 0.23 & 2.94 \\
\hline \multicolumn{4}{|c|}{ Fertilización química } \\
\hline Fert-0.5 & $2.64 b$ & $0.98 b$ & $30.91 \mathrm{ab}$ \\
\hline Fert-1.0 & $2.64 b$ & $1.32 \mathrm{a}$ & $29.94 b$ \\
\hline Fert-1.5 & $2.74 \mathrm{a}$ & $1.37 \mathrm{a}$ & $31.06 \mathrm{a}$ \\
\hline DSH (0.05) & 0.09 & 0.09 & 1.07 \\
\hline
\end{tabular}

Medias con letras iguales en las columnas no son estadísticamente diferentes (Tukey, 0.05), Sol: Solep, Fer: Fernatol, EV: estiércol vacuno, EO estiércol ovino, Fert: fertilización a 0.5, 1.0 y $1.5 \mathrm{~g} \mathrm{~L}^{-1}$, a: clorofila a, b: clorofila b, T: clorofila total, DSH: diferencia significativa honesta

González R., G. González, I. Acevedo, M. E. González y J. Contreras (2014) Producción de plántulas de pimentón (Capsicum annum L.) con sustrato a base de cachaza compostada. Revista de la Facultad de Agronomía (Luz) Supl. 1:182-190.

Guzmán M. and A. Sánchez (2003) Influence of nitrate and calcium increments on development, growth and early yield in sweet pepper plants. Acta Horticulturae 609:207-211, https://doi org/10.17660/ActaHortic.2003.609.29

Jayasinghe G. Y., I. D. L. Arachchi and Y. Tokashiki (2010) Evaluation of containerized substrates developed from cattle manure compost and synthetic aggregates for ornamental plant production as a peat alternative. Resources, Conservation and Recycling 54:1412-1418, https://doi.org/10.1016/j. resconrec.2010.06.002

Konica Minolta (1989) Chlorophyll meter SPAD-502 Instruction manual. Konica Minolta Sensing, Inc. Tokyo, Japan. 24 p.

López-Baltazar J., A. Méndez-Matías, L. Pliego-Marín, E. Aragón-Robles y M. L. Robles-Martinez (2013) Evaluación agronómica de sustratos en plántulas de chile 'onza' (Capsicum annuum) en invernadero Revista Mexicana de Ciencias Agrícolas 6:1139-1150, https:// doi.org/10.29312/remexca.v0i6.1278

Ortega-Martínez L. D., J. Sánchez-Olarte, R. Díaz-Ruiz y J. Ocampo-Mendoza (2010) Efecto de diferentes sustratos en el crecimiento de plántulas de tomate (Lycopersicum esculentum Mill). Ra Ximhai 6:365-372.

Piña M. y M. E. Arboleda (2010) Efecto de dos ambientes lumínicos en el crecimiento inicial y calidad de plantas de Crescentia cujete. Bioagro 22:61-66.

Reyes-Reyes J., A. Aldrete, V. M. Cetina-Alcalá y J. López-Upton (2005) Producción de plántulas de Pinus pseudostrobus var Apulcensis en sustratos a base de aserrín. Revista Chapingo Serie Ciencias Forestales y del Ambiente 11:105-110.

Rincón C. Á. y G. A. Ligarreto (2010) Relación entre nitrógeno foliar y el contenido de clorofila, en maíz asociado con pastos en el Piedemonte Llanero colombiano. Revista Corpoica - Ciencia y Tecnología Agropecuaria 11:122-128, https//doi.org/10.21930/ rcta.vol11_num2_art:202

Rodríguez M. R., E. G. Alcantar G., G. Iñiguez C., F. Zamora N., P. M. García L., M. A. Ruiz L. y E. Salcedo P. (2010) Caracterización física y química de sustratos agrícolas a partir de bagazo de agave tequilero Interciencia 35:515-520.

Romero-Arenas O., R. López E., M. A. Damián H., I. Hernández T., J. F. Parraguirre L. y M. Huerta L. (2012) Evaluación del residuo de cáscara de nuez (Juglans regia L.) en la producción de plántulas de Pinus patula, en vivero. Agronomía Costarricense 36:103-110.

Sáenz R. J. T., F. J. Villaseñor R., H. J. Muñoz F., A. Rueda S. y J. A. Prieto R. 
(2010) Calidad de planta en viveros forestales de clima templado en Michoacán. Folleto Técnico 17. Campo Experimental Uruapan. INIFAP. Uruapan, Michoacán, México. 48 p. SAS Institute, (2013) Base SAS® 9.4 Procedures Guide. Statistical Procedures. Second edition. Cary, North Carolina, USA. 550 p.

Tuzel Y. and G. B. Oztekin (2017) Organic seedling production. Acta
Horticulturae 1170:1141-1148, https://doi.org/10.17660/ ActaHortic.2017.1170.147

Tuzel Y., G. B. Oztekin and E. Tan (2014) Use of different growing media and nutrition in organic seedling production. Acta Horticulturae 1107:165-175, https://doi.org/10.17660/ ActaHortic.2015.1107.22 